\title{
MODELING, DESCRIPTION, AND CHARACTERIZATION OF FRACTAL PORE VIA MATHEMATICAL MORPHOLOGY
}

\author{
LAY LIAN TEO AND B. S. DAYA SAGAR \\ Received 3 February 2006; Accepted 29 March 2006
}

The aim of this paper is to provide description of fast, simple computational algorithms based upon mathematical morphology techniques to extract descriptions of pore channels_-throats_-and bodies and to represent them in 3D space, and to produce statistical characterization of their descriptions. Towards this goal, a model fractal binary pore is considered and is eroded recursively to generate different slices possessing decreasing degrees of porosity. By employing simple morphology-based approach, each slice of this pore space is decomposed into pore-channel, pore-throat, and pore-body, which are abstract structures that summarize the overall connectivity, orientation, and shape of the pore space. We consider the pore slices and their corresponding morphological quantities to stack them to further represent them in $3 \mathrm{D}$ space. We further provide a formulation essentially based on set theory to represent these three morphologic quantities to connect them appropriately across slices. The connected quantities are further fragmented to designate each fragmented portion with orders ranging from 1 to $\mathrm{N}$.

Copyright (c) 2006 L. L. Teo and B. S. D. Sagar. This is an open access article distributed under the Creative Commons Attribution License, which permits unrestricted use, distribution, and reproduction in any medium, provided the original work is properly cited.

\section{Introduction}

Heterogeneous material is one that is composed of domains of different materials (phases). In nature and man-made environment, examples of heterogeneous media, to name a few, include polycrystals, soils, sandstone, granular media, earth crust, sea ice, wood, bone, lungs, blood, animal and plant tissues, cell aggregates, and tumors [21]. The physical phenomenon of interest occurs on microscopic length scales (e.g., geological media that span from tens of nanometers to meters). Generally, structure on the microscopic length scale is referred to as microstructure. These microstructures can be characterized only statistically. Porous medium (e.g., sandstone porous medium obtained via microtomography) is one of such complex microstructures, the quantitative characterization of which is addressed in this investigation. Separation of rock matrix from its complimentary zone usually called porous phase, leaves an option to efficiently characterize porous 
phase. It is evident from the recent works on Fontainebleau sandstone that the characteristics derived through computer-assisted mapping and computer tomographic analysis were well correlated with the physical properties such as porosity, permeability, and conductance. Pore-phase forms due to random processes of grain deposition, cementation, and secondary thermal and geochemical processes. Sedimentalogical processes and subsequent modifications during transport and deposition control the morphology of porous medium. The physically viable reasons for observing any kind of geometry modification in the spatial organization of porous medium (whether the complex porous medium is transformed into simple porous medium or vice versa) include (a) fluid intrusion and extrusion in a nonlinear fashion, (b) encroachment of rock matrix into porephase, and (c) several chemical, biological, and mechanical forces acting internally and externally. Whatever the physical processes involved in altering the porous phase of material, we emphasized in this paper quantifying the complexity of porous phase in both $2 \mathrm{D}$ and $3 \mathrm{D}$ domains.

Pore morphologic description is an important part of many petrophysical systems. The classics of pore structure research $[2,7,13,20]$ recognized the importance of geometric analysis in understanding fluid transport through porous medium and derived relationships between the geometric properties of pore structure and the bulk properties of the medium. In recent past, retrieval of effective parameters from disordered heterogeneous material (e.g., porous media) has been done based on direct measurements, empirical relations as well as theoretical methods (see $[5,7,13,17,21]$ and the references therein). Evaluating morphological quantities remains subjective, unless these features are extracted and quantified. To date, quantifying techniques for extracting essential pore morphological quantities such as pore-channel, pore-throat, and pore-body rely on cumbersome approaches [7]. Retrieval of high-order information such as lineal path functions [12], chord length distribution [13, 22], pore-size distribution functions [16], coarseness [11], and autocorrelation functions [4] is important in determining a variety of effective properties [5]. To understand the transfer properties of the material, one should also introduce connectivity network models [7, 24], which are large-scale idealizations of the complex geometry of the porous media. The challenge in applying pore network models to rock is the characterization through geometrical descriptors and scaling through analysis of a pore space $[1,14,15]$. Turcotte [23] described a scaleinvariant sequential fragmentation process, which leads to a power-law number-size distribution of fragments. For a homogeneous structural model, notable relationships are derived through network models [3], which facilitate characterization of porous medium by means of pore coordination number, pore-channel, and pore-throat distributions. For a more realistic description of a pore network, different researchers used pore-throat length distribution, pore-body size distribution, throat-body size distribution, connectivity, and the spatial correlation between pore-bodies and pore-throats [3,7-10]. Pore size distribution can be obtained by a series of erosion-dilation algorithm [24]. Of late, several attempts have been made via image processing essentially mathematical morphological transformations as possible alternative approaches to isolate morphological quantities and further to characterize porous medium to relate with bulk properties [18]. We employed morphologic transformations in our earlier studies [19] for decomposing pore 
structure into nonoverlapping disks of various sizes and shapes, and this provided a fair representation and description of pore image morphology.

Mathematical morphology-based approaches have shown promising potential for porous medium description due to the fact that the mathematical morphology focuses on the geometric character of pore space. The three significant morphological quantities are extracted from complex pore space by representing it as an algebraic combination of number of components (e.g., channel, throat, and body), where each component is represented by combinations of channel, throats, and body subsets. It is intuitively true that the intricacy in the spatial pattern of these morphological features is the result of complex pore morphology itself. To a large extent, these morphological quantities determine rock porosity and permeability. In order to derive the relevant morphological quantities from porous medium, one needs high-resolution pore images and three-dimensional information.

In the present paper, to demonstrate the following three-step approach, we consider a Koch triadic fractal in binary form, and iteratively erode it with viable assumptions to generate model pore-slices and further to form a 3D fractal binary pore by stacking the pore-slices.

(i) The three significant geometric and/or topologic components describing organization of porous medium include (a) pore-channel, (b) pore-throat, and (c) pore-body. We employ mathematical morphology transformations and certain logical operations to isolate the three significant components from the binary pore-phase.

(ii) We proposed a way to extend an approach to map similar features from 3D pore-phases. This approach is primarily based on properly connecting the porechannel, -throat, -body subsets from respective slices of pore-phase.

(iii) We proposed scheme to designate orders for decomposed pore-bodies of various slices which has been subsequently taken as the basis to designate orders for both pore-channels and -throats. Once these components are designated appropriately with orders, we compute certain complexity measures for porous phase. Fractal characterization of pore-channel network and pore-body networks is carried out, and such an analysis facilitates complexity measures.

The information that follows includes basic transformations and material employed in Section 2, a simple mathematical framework to extract the three significant morphological quantities from pore-space and their representation in $3 \mathrm{D}$ space, respectively, in Sections 3 and 4. In Section 5, the results and the morphological relationships between the three quantities are discussed and finally concluded in Section 6.

\section{Methods and materials}

2.1. Methods. Morphological operations [17] such as erosion to shrink, dilation to expand, and opening and closing to smoothen are employed to transform $M$ into new images using a symmetric structuring element $S$ that acts as a probing rule with certain characteristic information such as shape, size, origin, and orientation. The essence of these transformations is illustrated by Figure 2.1. Morphologic erosion and dilation of $M$ with respect to $S$ are forms of local minima and maxima filters, which are usually denoted 
as $M \ominus S$ and $M \oplus S$ in (2.1) and (2.2),

$$
\text { erosion: }(M \ominus S)=\{m-s: m \in M, s \in S\}=\bigcap_{s \in S} M_{s} \text {. }
$$

To obtain eroded version, each pore element $\left(m_{i}\right)$ is translated with respect to $S$ of size one (Figure 2.1(a)) centered on $m_{i}$ to check whether all the elements in $S$ overlap with the neighboring elements of $m_{i}$. If exact overlap occurs, there would be no change required in the translate. Otherwise, the centered position in the image would be removed. Similarly, all other pore elements are translated by checking the nonoverlapping properties with reference to $S$. This erosion transformation is illustrated in matrix form (Figure 2.1(a)). In the erosion example, a $3 \times 3$ size $M$ is represented with 1's and 0 's that, respectively, stand for pore space and for rock-matrix regions. In $M$, five pore elements are obvious. These elements are systematically translated in terms of a symmetric $S$ with characteristic information of size $3 \times 3$ and rhombic shape, centered at the origin. For the case of erosion, each pore element in $M$ is systematically translated by means of $S$. The first translate is achieved in such a way that the origin in $S$ (i.e., the center point) is matched with the first encountered pore element of $M$ at location $(2,1)$. This location depicts the second column of the first scan line of $M$. Then we observe that $S$ is not in exact overlap with all adjacent pore elements. Hence, we consider this as "mismatch," and the first encountered pore element is transformed into a nonpore element. This is shown in the first translate involved in the erosion process. Similar translation is done for the second encountered pore element located at $(1,2)$ to check whether it exactly overlaps with $S$. As this second pore element also mismatches with reference to the origin of $S$, the second translate for pore element at location $(1,2)$ is transformed into nonpore element. Similar exercise provides five translates as shown in Figure 2.1(a). It is obvious that the translate achieved for the third encountered pore element at location $(2,2)$ exactly matches $S$. Hence, the corresponding translate is unique. Further, the intersection of all translates provides the eroded version of $M$ by $S$,

$$
\text { dilation: } M \oplus S=\{m+s: m \in M, s \in S\}=\bigcup_{s \in S} M_{s} \text {. }
$$

The rule followed to translate the pore elements to further achieve dilation is slightly different from the rule followed in the erosion process. While matching the first encountered pore element at location $(2,1)$ with reference to the center point of $S$, we check for exact overlap with all points in $S$ with all pore elements. As for the first encountered pore element, we see that there is a mismatch. Then the points of $S$ that are not exactly matched with pore elements would be placed at locations beyond the pore elements. This can be better comprehended from the first translate shown in Figure 2.1(b). Similarly, the second and the further translates are shown. As at the third encountered pore element the matching is exactly identified by means of $S$, its translate is unique. The union of all these translates produces the dilated version of $M$ by $S$. The number of translates required to achieve either erosion or dilation (Figures 2.1(a) and 2.1(b)) is equivalent to the number of pore elements. Hence, five translates are required, for the examples shown in Figures 2.1(a) and 2.1(b), each for erosion and dilation. Implementation of erosion and dilation 


\begin{tabular}{|c|c|c|c|c|c|c|}
\hline (a) Morphological erosion of $M$ by $S$ \\
\hline
\end{tabular}

(b) Morphological dilation of $M$ by $S$

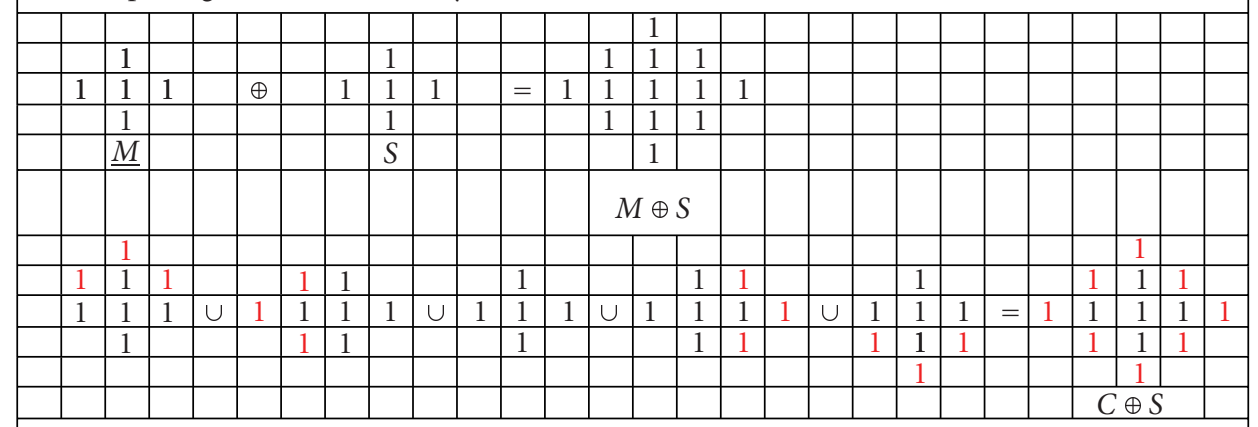

(c) Morphological opening of $M$ by $S$

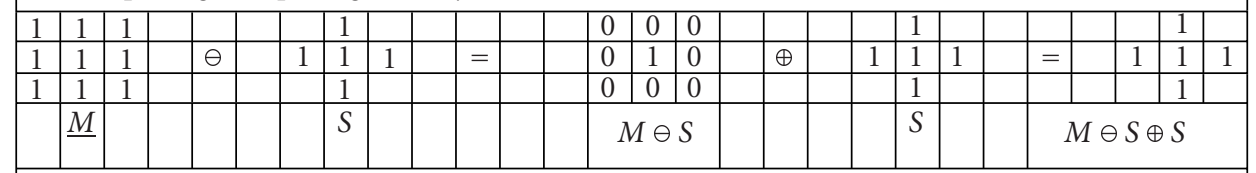

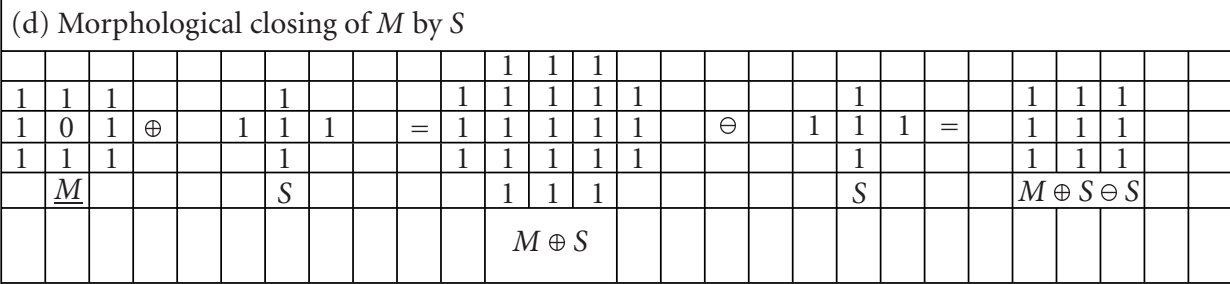

(e) Minkowski addition of structuring templates

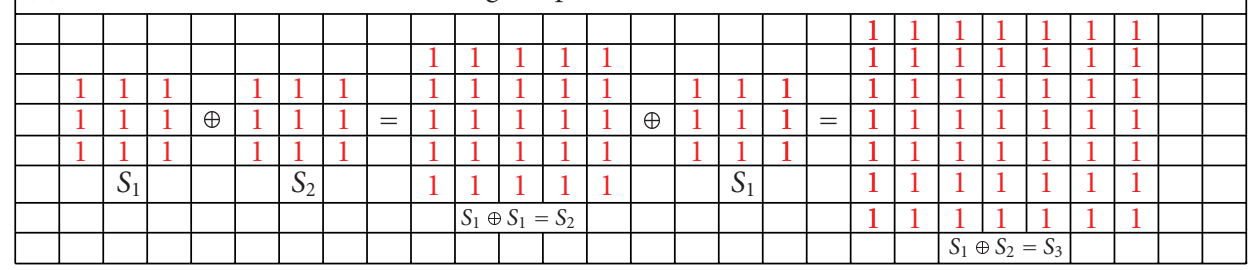

Figure 2.1. Matrix representation of morphological transformations and certain logical operations. These transformations are performed with the help of $S$ centered on $m$.

transformations as cascades of erosion-dilation and dilation-erosion yields, respectively, the secondary opening and closing operations, denoted as $[M \ominus S \oplus S]$ and $[M \oplus S \ominus S]$ 


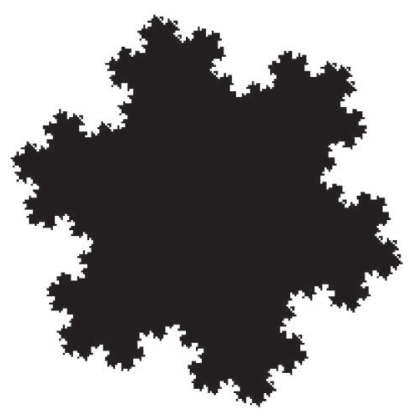

Figure 2.2. Model fractal binary pore.

or simply $M \circ S$ and $M \bullet S$ in (2.3) and (2.4),

$$
\text { opening: } M \circ S=(M \ominus S) \oplus S \text {. }
$$

Opening transformation is illustrated in Figure 2.1(c), where a cascade of erosion followed by dilation of $M$ with nine pore elements with respect to $S$ is shown. To perform erosion first on the nine pore elements, nine translates are required. Then the resultant eroded version is dilated to achieve the opened version of $M$ by $S$ as shown in Figure 2.1(c),

$$
\text { closing: } M \bullet S=(M \oplus S) \ominus S \text {. }
$$

Morphological closing transformation is illustrated in Figure 2.1(d). To achieve closed version of $M$ by $S$ (Figure 2.1(d)), we first perform dilation on $M$ of size $3 \times 3$ with nine pore elements using $S$, followed by erosion on the resulting dilated version. We employ recursive erosions and dilations to perform multiscale opening. In the multiscale opening, a cascade of erosion-dilation can be performed with respect to the structuring element $S$ with scaling factor $n$. In this approach, the size of the structuring template increases from iteration to iteration as

$$
n S=\underbrace{\mathbf{s} \oplus \mathbf{s} \oplus \mathbf{s} \oplus \cdots \oplus \mathbf{s}}_{n \text { times }}
$$

structuring templates of varied sizes and their Minkowski sums. To perform the transformations shown in Figures 2.1(a)-2.1(d), by changing the scale of $S$, one requires taking the addition of $S$ by $S$ to a desired level (2.5). As an example, we show in Figure 2.1(e) how we get $S_{2}$ by adding $S_{1}$ with $S_{1}$.

2.2. Materials. We consider a fractal binary pore of size $256 \times 256$ pixels (Figure 2.2) with $33 \%$ porosity level. We generate different slices of this model pore by iteratively eroding till it vanishes. This pore space took 28 iterative erosions with respect to octagon structuring element of primitive size of $5 \times 5$. With the assumption already mentioned, we stack the 55 slices to form the fractal pore in $3 \mathrm{D}$. Twenty-seven erosion cycles are performed on fractal binary pore (Figure 2.3) to vanish completely. 


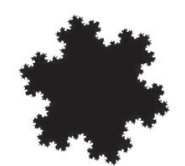

(a)

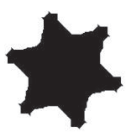

(f)

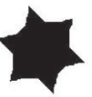

(k)

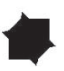

(p)

(u)

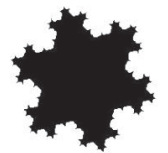

(b)

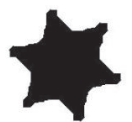

(g)

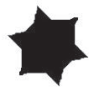

(1)

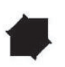

(q)

(v)

(z)

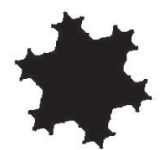

(c)

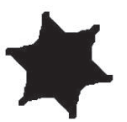

(h)

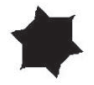

(m)

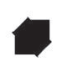

(r)

(w)

(za)

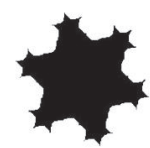

(d)

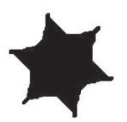

(i)

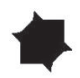

(n)

(s)

$(\mathrm{t})$

(x)

(y) (o)

(e)

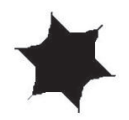

(j)

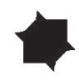

(zb)

Figure 2.3. Fractal pore under increasing cycles of erosion transformation by octagon structuring element. 


\section{Decomposition of the pore space to pore-channel, -throat, and -body}

Pore-bodies, pore-throats, and pore-channels are defined as follows: larger pore space openings in a fluid-bearing rock, where most of the fluid is stored, are pore-bodies and narrow gateways that connect the pore-bodies of various sizes (orders) are pore-throats. The channel connecting all the centers of major pore-bodies of various sizes that are approximated by regular components (structuring elements) is termed as pore-channel. One can see the illustrative meanings of these definitions from Figure 3.1. In 2D, a structure similar to that shown in Figure 3.1, according to these definitions, there exist 4 orders of pore-bodies (in nonoverlapping way), pore-throats, and pore-channels. Orders ranging from 1 to $n$ in Figure 2.2 are illustratively shown for pore-channels, -throats, and -bodies in 2D case. Their extensions to the 3D space are shown in (4.7)-(4.11) and in model fractal pore. More information about the scheme followed for order designations can be seen in the fourth section. To decompose the pore space into channels, throats, and pore-bodies of various lengths and sizes, we employ both primary and secondary morphologic transformations combined with certain logical operations and skeletonization $[6,17]$. Morphological skeletonization, shape decomposition, and morphological reconstruction from skeleton procedures are employed to retrieve three significant pore morphological quantities, namely, pore-channel, -throat, and -body networks from twodimensional pore space [19]. Similar approach has been employed on the sequentially eroded fractal binary pore object. We employ mathematical morphological transformations to decompose a model fractal pore into pore-connectivity, -throat, and -body.

The medial axis [18] of the pore-image is well known to represent a geometrically, connected, descriptor of the centerline skeleton of any porous medium. The PCNs (Figure 3.2) obtained here for a $2 \mathrm{D}$ slicewise pore-images (e.g., Figures 3.2(a)-3.2(z)) are composed of a union of connected subsets of the corresponding medial axes.

3.1. Pore-channel network. Pore-channel of a $2 \mathrm{D}$ slice, $\left[\mathrm{PCN}\left(M^{j}\right)\right], j=1,2, \ldots, N$, is the network obtained by connecting the loci of all maximal inscribable disks in the pore. To obtain PCN subsets of order $n$, we subtract the opened $n$ th-level eroded version from the pore that is eroded to $n$th level. This process is depicted as

$$
\operatorname{PCN}_{n}\left(M^{j}\right)=\left(M^{j} \ominus n S\right) /\left[\left(M^{j} \ominus n S\right) \circ S\right], \quad n=0,1, \ldots, N, j=1,2, \ldots, N,
$$

where $\left[\mathrm{PCN}_{n}\left(M^{j}\right)\right]$ denotes the $n$th pore network subsets of $j$ th slice of pore object points having maximal inscribable disks of size $n, n S$ is a template of size $n$. PCN subsets of increasing order, $\left[\mathrm{PCN}_{n}\left(M^{j}\right)\right]$ with $n=0,1,2, \ldots, N$, are isolated by performing morphologic erosions with structuring elements $S$ of increasing size and then performing opening on the resultant eroded version. To extract pore-channel network (PCN) subsets of order $n$ (3.1), we perform opening by an arbitrary size structuring element. In the above expression, subtracting the opened eroded version of $M$ from the eroded version by $n S$ retains only the angular points, which are the pore-channel subsets. The subtraction is achieved by a simple logical operation, represented by the symbol ( $\backslash$ ) in (3.1). The union 

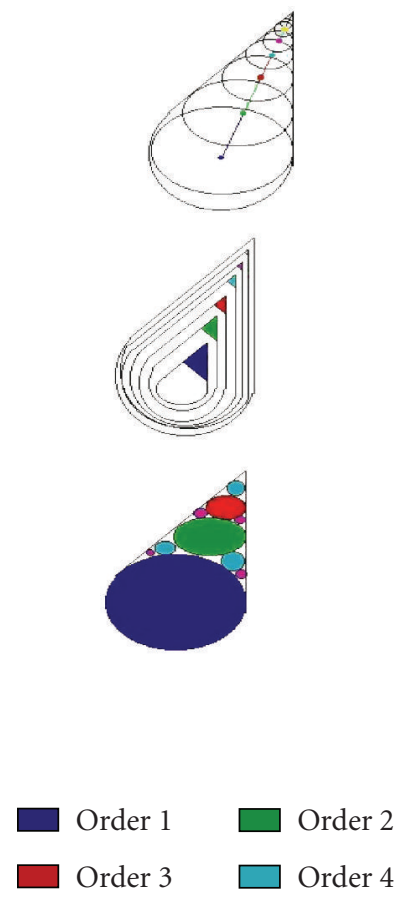

Figure 3.1. Schematic representations of pore-channel, -throat, and -body.

of all possible PCN subsets produces $\operatorname{PCN}\left(M^{j}\right)(3.2)$,

$$
\operatorname{PCN}\left(M^{j}\right)=\bigcup_{n=0}^{N} \operatorname{PCN}_{n}\left(M^{j}\right) .
$$

3.2. Pore-throat network. The PTN is obtained (Figure 3.3) as the union of intersectional portions of reconstructed channel subsets. The throat sizes are in increasing order from the pore-grain interface to the main pore-body. We choose an $n$-size structuring element to perform opening in (3.1), the operation would yield an $n$-size pore-throat (3.3),

$$
\operatorname{PTN}_{n}\left(M^{j}\right)=\left(M^{j} \ominus n S\right) /\left[\left(M^{j} \ominus n S\right) \circ n S\right], \quad n=0,1, \ldots, N ; j=1,2, \ldots, N .
$$

The union of $n$th size pore-throats provides pore-throats of all existing sizes in the pore structure as shown in (3.4),

$$
\operatorname{PTN}\left(M^{j}\right)=\bigcup_{n=0}^{N} \operatorname{PTN}_{n}\left(M^{j}\right) .
$$


10 Modeling and analysis of porous phase

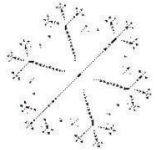

(a)

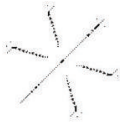

(f)

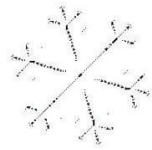

(b)

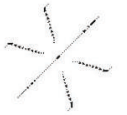

(g)

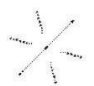

(l)

(k)<smiles>CC1(C)CC1(C)C</smiles>

3

(p)

(q)

(v)

(u)

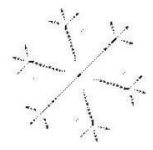

(c)

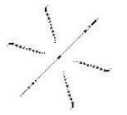

(h)

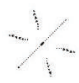

(m)

(r)<smiles>CC1(C)CC1</smiles>

(n)

(o) (e)

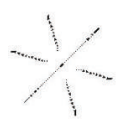

(i)

(j) (s)

(t)

(x)

(y)

(z)

(za)

(zb)

Figure 3.2. Pore-channel network from eroded versions of fractal pore. 
L. L. Teo and B. S. D. Sagar 11

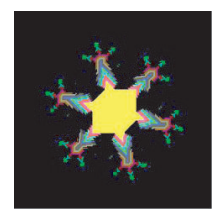

(a)

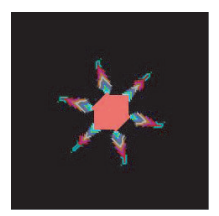

(f)

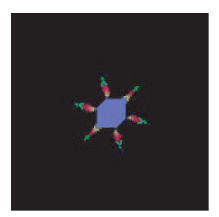

(k)

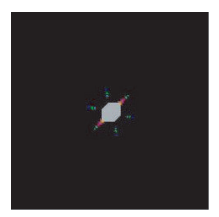

(p)

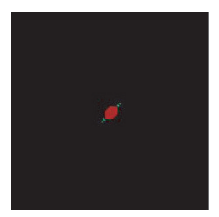

(u)

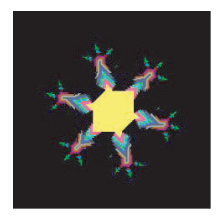

(b)

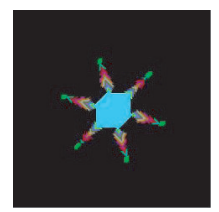

(g)

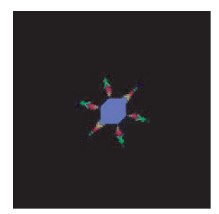

(l)

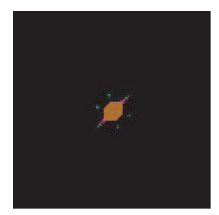

(q)

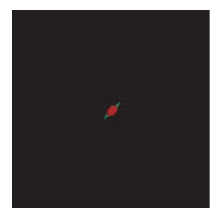

(v)

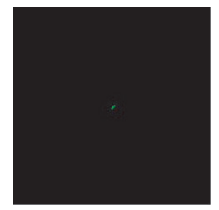

(z)

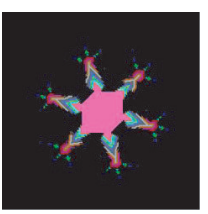

(c)

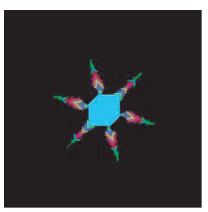

(h)

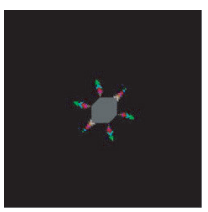

(m)

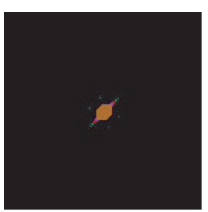

(r)

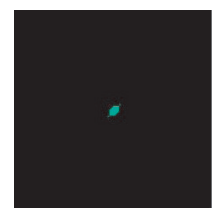

(w)

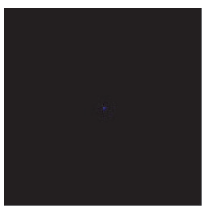

(za)

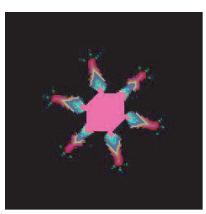

(d)

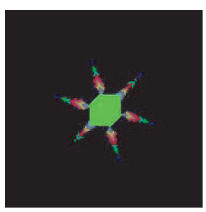

(i)

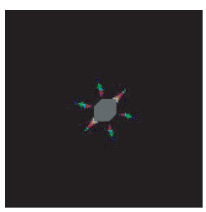

(n)

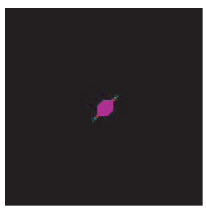

(s)

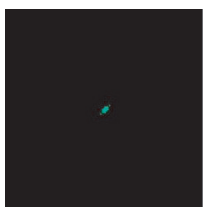

(x)

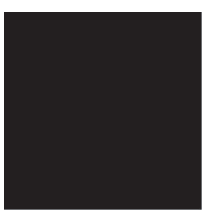

(zb)

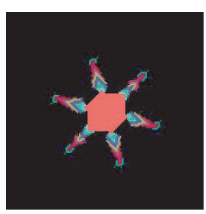

(e)

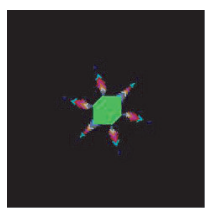

(j)

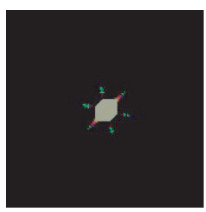

(o)

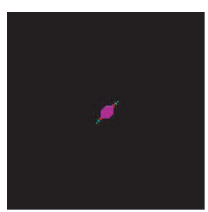

(t)

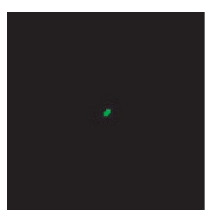

(y)

Figure 3.3. Pore-throat network from eroded versions of fractal pore. 
Each slice of fractal binary pore is transformed as pore-throats of various sizes. A sequence of pore-throat network is shown (Figure 3.3), in which, for better legibility, colorcoding is done.

3.3. Pore-body network. The PBN is analogous to the maximal balls [18]. The PBN of pore is obtained via decomposition of $2 \mathrm{D}$ pore space into nonoverlapping bodies of different and well-defined sizes. A procedure including a multiscale opening and certain logical operators is employed to decompose a pore structure into pore-bodies of various sizes. After performing $n$ times the multiscale opening, the resulting pore space needs to be subtracted from the original version. While multiscale opening with respect to $S$ of size $N+1$ eliminates the pore space, multiscale opening by an $S$ of size $N$ needs to be performed to decompose the main pore space, and its successively obtained subtracted portions. By taking into account the condition that $N+1$ iterations of multiscale opening eliminate the respective level of pore space or the successively subtracted portions, each subtracted portion is subjected to further decompositions. Here, the size $N$ to perform multiscale opening depends on the size and shape of the subsequently subtracted pore-images. The number of subtracted portions that may appear while decomposing a pore space depends on its size and the shape, as well as on the structuring template. Symbolically, the morphological decomposition procedure is given by (3.5) and (3.6),

$$
M_{i+1}^{j}=\left\lfloor M_{i}^{j} / M_{i}^{j} \circ N S\right\rfloor,
$$

where $i=0,1,2, \ldots, N, j=1,2, \ldots, N ; M_{i+1} \subset M_{i}$,

$$
\operatorname{PBN}\left(M^{j}\right)=\bigcup_{i=0}^{N}\left(M_{i}^{j} o N S\right) .
$$

Implementing these sequential steps by means of an octagon, we obtain Figures 3.4(a)$3.4(\mathrm{zb})$. Each eroded fractal binary pore is decomposed into nonoverlapping octagons of different sizes. With the progressive shrinking, it is obvious that the number of octagon category (categories) and its (their) size (sizes) are also reduced. Each order of decomposed category(ies) is color-coded for better legibility. The characteristic information of considered primitive structuring element includes octagonal shape, size of $5 \times 5$ pixels, origin at 3, 3, coordinate positions, and symmetric orientation. Change in size of this template shows impact in terms of scale change. Changes in other characteristic information show implications with changes in the geometric and spatial organizations of pore-channels, -throats, and -bodies. Since we adopted octagonal element that is symmetric about origin, the spatial organization of fractal pore that is symmetric from left to right and from top to bottom yields rather regular spatial organization of these features. The procedure results in size distributed pore-channel, -throats, and -bodies that further facilitate the characterization of pore morphologic complexity. These transformations could also be the bases for further analyses of connectivity while the extensions to the three dimensions should be obvious. The use of these three transformations to retrieve significant pore morphologic quantities is further shown to visualize a $3 \mathrm{D}$ fractal binary pore-channel, -throat, and -body. Based on the procedures in the previous section, using 


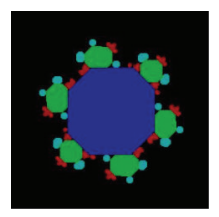

(a)

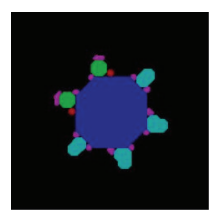

(f)

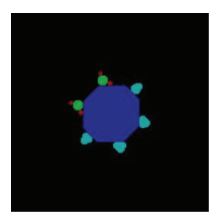

(k)

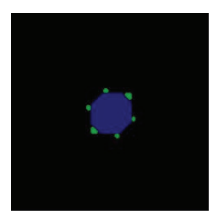

(p)

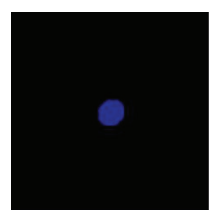

(u)

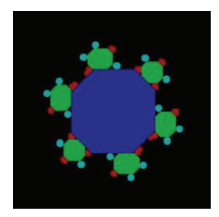

(b)

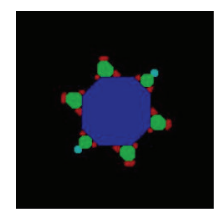

(g)

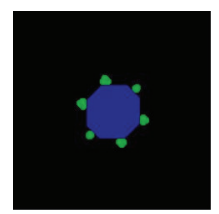

(1)

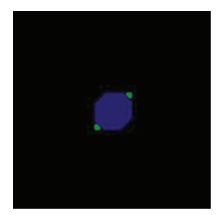

(q)

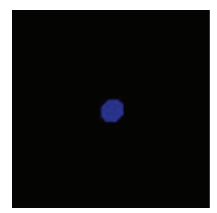

(v)

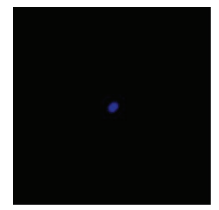

(z)

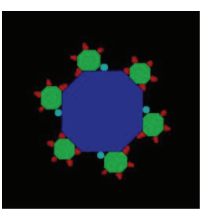

(c)

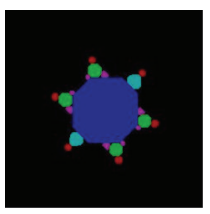

(h)

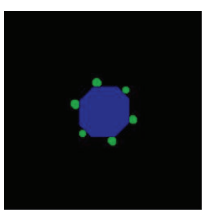

(m)

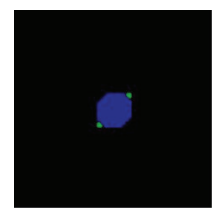

(r)

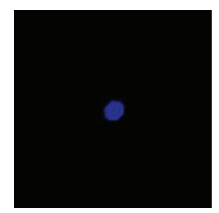

(w)

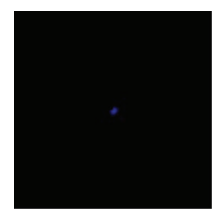

(za)

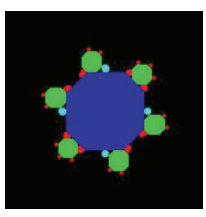

(d)

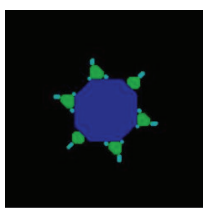

(i)

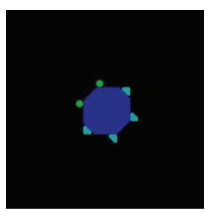

(n)

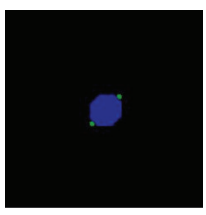

(s)

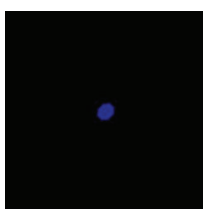

(x)

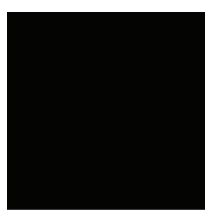

(zb)

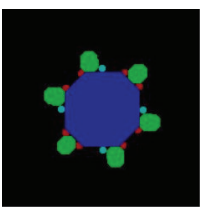

(e)

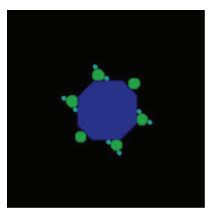

(j)

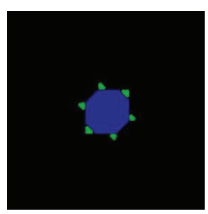

(o)

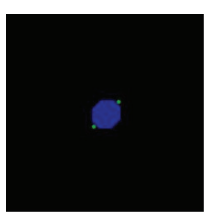

(t)

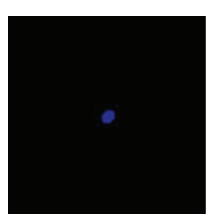

(y)

Figure 3.4. Pore-body network from eroded versions of fractal pore. 
an octagonal structuring element, we decompose the pore structure into pore-channels, -throats, and -bodies (Figures 3.2-3.4). For better perception, each level of the decomposed pore-throats and pore-bodies is color-coded (Figures 3.3 and 3.4).

\section{Visualization of pore-channel, -throat, and -body networks in 3D space: a fractal binary pore}

These recursively eroded pore-phases to different degrees are considered as slices and a $3 \mathrm{D}$ fractal pore is constructed systematically. Sequentially eroded versions (Figure 2.3) of fractal pore model are used to represent a 3D fractal pore model. In order to visualize the existing pore connectivity, -throats, and -body networks in 3D space, each eroded slice in $2 \mathrm{D}$ space is parallely considered to extract slicewise pore connectivity, -throat, and -body networks. Furthermore, such information decomposed from all slices is considered to visualize their three-dimensional forms. And such 2D pore features are stacked to construct pore-connectivity, -throat, and -body network in three-dimensional space. $N$ th-level decomposed channel, throat, and body subset(s) of each pore slice are used to construct $N$ th-level 3D decomposed channel, throat, and body.

4.1. 3D fractal binary pore. We form the $3 \mathrm{D}$ fractal pore as per the scheme shown in the schematic representation in Figure 4.1. In the stack, original fractal binary pore (Figure 2.2) is embedded at the middle and superposed on both sides of it with the fractal binary pore slice generated by performing increasing cycles of erosion transformation. Each eroded version is superposed on one another as shown in the figure to reconstruct $3 \mathrm{D}$ fractal binary pore. In turn, in the stack of 3D pore image, top and bottom most pore slices possess less porosity $(0.019836 \%)$ followed by immediate inner slices with porosity $(0.093079 \%)$ and so on (Table 4.1$)$. The middle slice in the stack possesses the porosity of $32.49512 \%$. The reason for inserting the pore slices on top and bottom of the middle slices is only to illustrate the model with symmetry. The thickness of each slice is computed as one voxel. Hence the $3 \mathrm{D}$ fractal pore data is with the specifications of $256 \times 256 \times 55$. Stack of the pore-image slices is represented by the set

$$
G=\left\{M^{1}, M^{2}, \ldots, M^{N}\right\}
$$

where $N$ is the total number of pore-image slices in the stack. For the present case, $N$ is considered as 55 . We denote each pore-image slice by $M^{j}$, where $j$ is the slice index. Entire stack of such pore-image slice is depicted by the set $G$. We followed similar scheme to stack pore object, pore-channel, -throat, and -body further to represent them in $3 \mathrm{D}$ with different views (Figure 4.2).

4.2. 3D pore-channel, -throat, and -body, and their fragmentation. Stack of the porechannel of various orders ranging from 0 to $N$ decomposed, respectively, from each poreimage slice is represented by the set

$$
\operatorname{PCN}(G)=\left\{\left[\operatorname{PCN}^{1}(G)\right],\left[\operatorname{PCN}^{2}(G)\right], \ldots,\left[\operatorname{PCN}^{N-1}(G)\right],\left[\operatorname{PCN}^{N}(G)\right]\right\}
$$




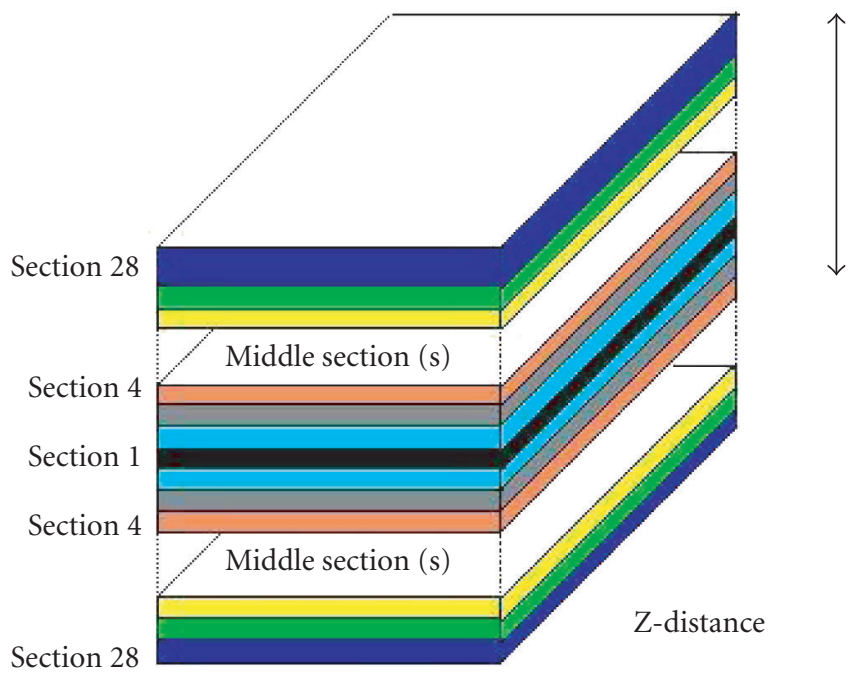

Figure 4.1. Schematic of construction of 3D pore and morphological quantities. For pore sections 1 to 28 , iteratively eroded pore slices shown in Figures $2.3(\mathrm{a})-2.3(\mathrm{zb})$ are considered to form $3 \mathrm{D}$ pore (Figures 4.2(a)-4.2(b)).

where

$$
\begin{gathered}
\operatorname{PCN}^{1}(G)=\left\{\left[\operatorname{PCN}_{N}\left(M^{1}\right)\right],\left[\operatorname{PCN}_{N}\left(M^{2}\right)\right], \ldots,\left[\operatorname{PCN}_{N}\left(M^{N-1}\right)\right],\left[\operatorname{PCN}_{N}\left(M^{N}\right)\right]\right\}, \\
\operatorname{PCN}^{2}(G)=\left\{\left[\operatorname{PCN}_{N-1}\left(M^{1}\right)\right],\left[\operatorname{PCN}_{N-1}\left(M^{2}\right)\right], \ldots,\left[\operatorname{PCN}_{N-1}\left(M^{N-1}\right)\right],\right. \\
\left.\left[\operatorname{PCN}_{N-1}\left(M^{N}\right)\right]\right\} \\
\vdots \\
\operatorname{PCN}^{N}(G)=\left\{\left[\operatorname{PCN}_{1}\left(M^{1}\right)\right],\left[\operatorname{PCN}_{1}\left(M^{2}\right)\right], \ldots,\left[\operatorname{PCN}_{1}\left(M^{N-1}\right)\right],\left[\operatorname{PCN}_{1}\left(M^{N}\right)\right]\right\} .
\end{gathered}
$$

The stack of all higher-order channel subsets extracted from each slice would form first-order pore-channel network in 3D, with a condition that the first-order channel network is contained in the first-order pore-body network. Similarly, the stack of secondorder pore-channel network is contained in the second-order pore-body network, and so on until the stack of $N$ th-order pore-channels is contained in the largest order pore-body network. In the present model, pore-channels of third order exist with only one slice. Hence these channel subsets posses no connectivity with that of other slices. Stack of the pore-throats of various orders ranging between 1 to $N$ extracted from each pore-image slice is represented by set

$$
\operatorname{PTN}(G)=\left\{\left[\operatorname{PTN}^{1}(G)\right],\left[\operatorname{PTN}^{2}(G)\right], \ldots,\left[\operatorname{PTN}^{N-1}(G)\right],\left[\operatorname{PTN}^{N}(G)\right]\right\},
$$


16 Modeling and analysis of porous phase

Table 4.1. Orderwise number of pixels at each slice of fractal pore-channel, -throat, and -body.

\begin{tabular}{|c|c|c|c|c|c|c|c|c|c|c|c|c|c|}
\hline \multirow{3}{*}{$\begin{array}{l}\text { Slice } \\
(N)\end{array}$} & \multirow{3}{*}{$\begin{array}{l}\text { Porosity } \\
\text { across } \\
\text { slices }\end{array}$} & \multirow{3}{*}{$\begin{array}{l}\text { Pore- } \\
\text { channel }\end{array}$} & \multirow{3}{*}{$\begin{array}{l}\text { Pore- } \\
\text { throat }\end{array}$} & \multirow{3}{*}{$\begin{array}{l}\text { Pore- } \\
\text { body }\end{array}$} & \multicolumn{9}{|c|}{ Orderwise pore-channels, -throats, and -bodies } \\
\hline & & & & & \multicolumn{3}{|c|}{ Channel } & \multicolumn{3}{|c|}{ Throat } & \multicolumn{3}{|c|}{ Body } \\
\hline & & & & & 1 & 2 & 3 & 1 & 2 & 3 & 1 & 2 & 3 \\
\hline 1 & 32.49 & 987 & 9601 & 21296 & 180 & 445 & 362 & 3279 & 3054 & 3066 & 11083 & 5046 & 3230 \\
\hline 2 & 27.9 & 682 & 8132 & 18303 & 180 & 439 & 57 & 2753 & 2570 & 2310 & 10309 & 4574 & 2167 \\
\hline 3 & 24.67 & 619 & 7454 & 16173 & 180 & 445 & - & 2753 & 2475 & 1630 & 9563 & 4120 & 1732 \\
\hline 4 & 21.96 & 475 & 6322 & 14396 & 180 & 295 & - & 2293 & 2058 & 1542 & 8845 & 3584 & 1126 \\
\hline 5 & 19.57 & 412 & 5679 & 12831 & 180 & 232 & - & 2293 & 1947 & 1135 & 8155 & 3213 & 726 \\
\hline 6 & 17.52 & 358 & 4883 & 11484 & 180 & 178 & - & 1890 & 1630 & 1032 & 7493 & 2669 & 854 \\
\hline 7 & 15.65 & 342 & 4387 & 10262 & 180 & 162 & - & 1890 & 1519 & 718 & 6859 & 1932 & 1092 \\
\hline 8 & 13.92 & 336 & 3676 & 9128 & 180 & 156 & - & 1545 & 1238 & 629 & 6253 & 1602 & 862 \\
\hline 9 & 12.26 & 330 & 3182 & 8038 & 330 & - & - & 1545 & 1123 & 358 & 5675 & 1476 & 520 \\
\hline 10 & 10.65 & 246 & 2474 & 6982 & 246 & - & - & 1243 & 892 & 193 & 5125 & 1114 & 240 \\
\hline 11 & 920 & 210 & 2133 & 6048 & 210 & - & - & 1243 & 777 & 113 & 4603 & 985 & 102 \\
\hline 12 & 700 & 180 & 1692 & 5241 & 180 & - & - & 979 & 605 & 108 & 4109 & 780 & 0 \\
\hline 13 & & 145 & 1468 & 4510 & 145 & - & - & 979 & 406 & 83 & 3643 & 558 & 0 \\
\hline 14 & 0.00 & 129 & 1134 & 3855 & 129 & - & - & 751 & 357 & 26 & 3205 & 494 & 0 \\
\hline 15 & & 103 & 1000 & 3279 & 103 & - & - & 751 & 249 & - & 2795 & 388 & 0 \\
\hline 16 & & & & & 77 & - & - & 553 & 176 & - & 2413 & 252 & 0 \\
\hline 17 & 3.49 & 56 & 658 & 2293 & 56 & - & - & 553 & 105 & - & 2059 & 102 & 0 \\
\hline 18 & & & & & 43 & - & - & 385 & 80 & - & 1733 & 80 & 0 \\
\hline 19 & & 35 & & 1545 & 35 & - & - & 385 & 46 & - & 1435 & 60 & 0 \\
\hline 20 & 1.89 & & & 1243 & 27 & - & - & 247 & 40 & - & 1165 & 42 & 0 \\
\hline 21 & & 20 & & & 25 & - & - & 247 & 20 & - & 923 & 0 & 0 \\
\hline 22 & & 23 & & & 23 & - & - & 139 & 20 & - & 709 & 0 & 0 \\
\hline 23 & 0 & 2 & 147 & 553 & 21 & - & - & 139 & 8 & - & 523 & 0 & 0 \\
\hline 24 & & . & 69 & & 19 & - & - & 61 & 8 & - & 365 & 0 & 0 \\
\hline 25 & & 1 & 6 & & 17 & - & - & 61 & 2 & - & 235 & 0 & 0 \\
\hline 26 & ניר 0 & 1 & 15 & 130 & 15 & - & - & 61 & - & - & 133 & 0 & 0 \\
\hline 27 & & 13 & 13 & 61 & 13 & - & - & 13 & - & - & 59 & 0 & 0 \\
\hline 28 & 0.019 & 13 & 0 & 13 & 13 & - & - & - & - & - & 0 & 0 & 0 \\
\hline & Total & 5938 & 66520 & 1109016 & 3167 & 2352 & 419 & 28384 & 21405 & 12943 & 748263 & 219945 & 79459 \\
\hline
\end{tabular}




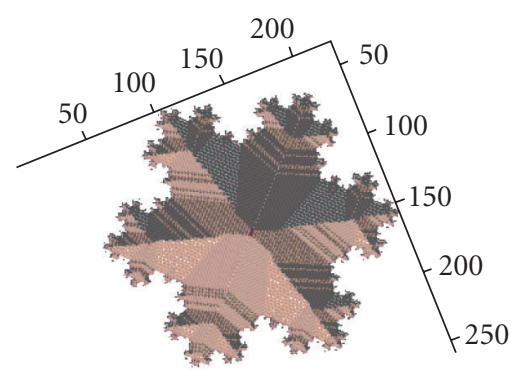

(a)

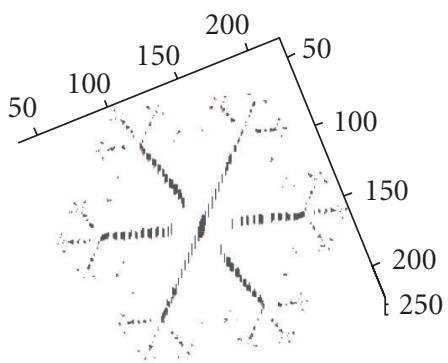

(c)

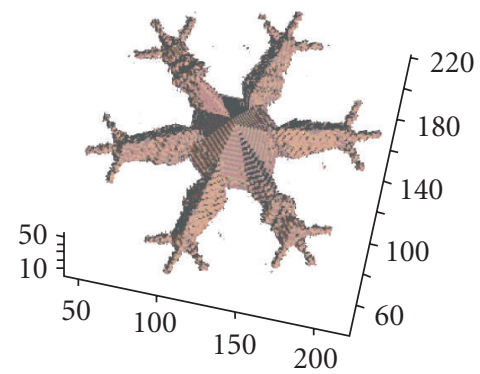

(e)

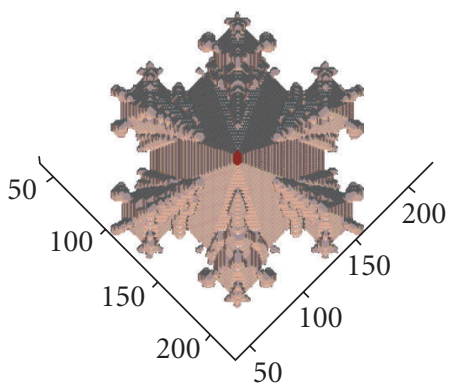

(g)

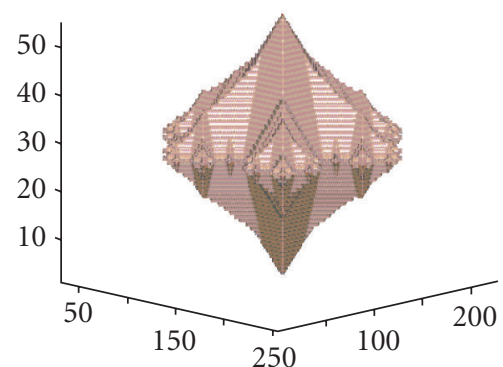

(b)

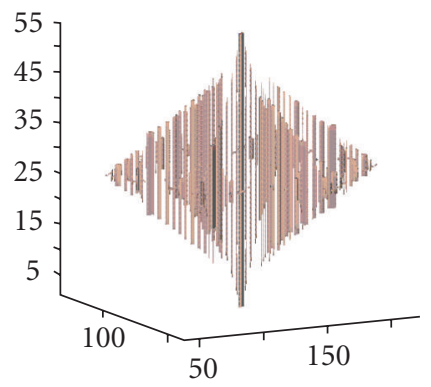

(d)

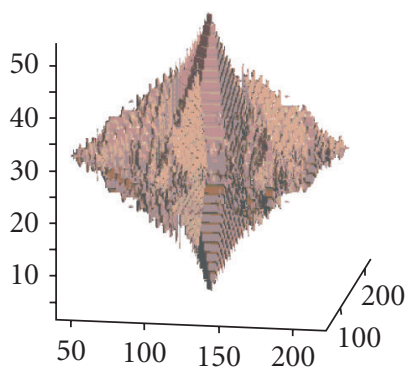

(f)

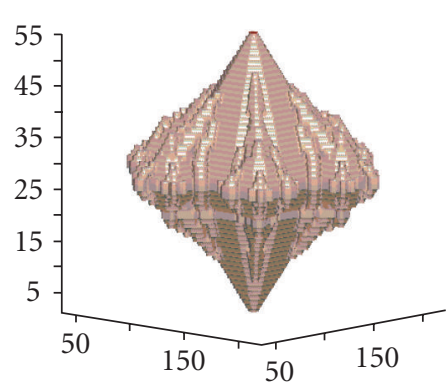

(h)

Figure 4.2. Top and side views of (a)-(b) model 3D fractal binary pore, (c)-(d) pore-channel, (e)-(f) pore-throat, and $(\mathrm{g})-(\mathrm{h})$ pore-body. 
where

$$
\begin{aligned}
& \operatorname{PTN}^{1}(G)=\left\{\left[\operatorname{PTN}_{N}\left(M^{1}\right)\right],\left[\operatorname{PTN}_{N}\left(M^{2}\right)\right], \ldots,\left[\operatorname{PTN}_{N}\left(M^{N-1}\right)\right],\left[\operatorname{PTN}_{N}\left(M^{N}\right)\right]\right\}, \\
& \operatorname{PTN}^{2}(G)=\left\{\left[\operatorname{PTN}_{N-1}\left(M^{1}\right)\right],\left[\operatorname{PTN}_{N-1}\left(M^{2}\right)\right], \ldots,\left[\operatorname{PTN}_{N-1}\left(M^{N-1}\right)\right],\right. \\
& {\left.\left[\operatorname{PTN}_{N-1}\left(M^{N}\right)\right]\right\} } \\
& \vdots \operatorname{PTN}^{N}(G)=\left\{\left[\operatorname{PTN}_{1}\left(M^{1}\right)\right],\left[\operatorname{PTN}_{1}\left(M^{2}\right)\right], \ldots,\left[\operatorname{PTN}_{1}\left(M^{N-1}\right)\right],\left[\operatorname{PTN}_{1}\left(M^{N}\right)\right]\right\} .
\end{aligned}
$$

The stack of higher-order throat subsets extracted from each slice would form firstorder pore-throat network in 3D. This first-order throat network is contained in the first-order pore-body network. Similarly, the stack of second order pore-channel network should be contained in the second-order 3D pore-body network, and so on. Similarly, stack of the pore-bodies of various orders ranging from 1 to $\mathrm{N}$ decomposed, respectively, from each slice of pore-image is represented by the set

$$
\operatorname{PBN}(G)=\left\{\left[\operatorname{PBN}^{1}(G)\right],\left[\operatorname{PBN}^{2}(G)\right], \ldots,\left[\operatorname{PBN}^{N-1}(G)\right],\left[\operatorname{PBN}^{N}(G)\right]\right\},
$$

where

$$
\begin{gathered}
\operatorname{PBN}^{1}(G)=\left\{\left(M_{1}^{1} \circ N S\right),\left(M_{1}^{2} \circ N S\right), \ldots,\left(M_{1}^{N-1} \circ N S\right),\left(M_{1}^{N} \circ N S\right)\right\}, \\
\operatorname{PBN}^{2}(G)=\left\{\left(M_{2}^{1} \circ N S\right),\left(M_{2}^{2} \circ N S\right), \ldots,\left(M_{2}^{N-1} \circ N S\right),\left(M_{2}^{N} \circ N S\right)\right\} \\
\vdots \\
\operatorname{PBN}^{N}(G)=\left\{\left(M_{N}^{1} \circ N S\right),\left(M_{N}^{2} \circ N S\right), \ldots,\left(M_{N}^{N-1} \circ N S\right),\left(M_{N}^{N} \circ N S\right)\right\},
\end{gathered}
$$

where superscript and subscript $N s$, respectively, denote index of pore-image slice and order of the decomposed pore-channel, (-throat, and -body), and NS denotes the size of structuring element. In the current fractal pore model, the pore-body network is designated with three different orders (Figures $4.3(\mathrm{e})-4.3(\mathrm{~g})$ ). The order of the decomposed pore-body can be determined by a recursive relationship shown in (3.5) and (3.6). The $N$ th-level decomposed body from all the slices ranging from $j=1,2, \ldots, N$ would be put in a separate stack, which is considered as first-order fragmented pore-body from $G$. Similarly, $N$ th-level decomposed pore-bodies, that are smaller than the $N$ th-level decomposed disks from the previous order, from the reminder of respective slices, are stacked to visualize the pore-bodies of second-order in $3 \mathrm{D}$. This is a recursive process, till all the Nthlevel decomposed pore-bodies of decreasing sizes that pore retrieval from different levels of each pore slice are stacked. The pore-channel and throats are segregated into first, second, and third orders in such as way that they are, respectively, contained in first-, second-, and third-order pore-body networks. In other words, the pore-channel subsets of varied orders are the centers of the pore-bodies of varied orders. If there exists no porechannel subset(s) or -throat(s), then there will be no pore-body(ies) existing. $N$ th-order pore-channel subset is a subset of $N$ th-order pore-bodies. Due to these facts, the volumes of pore-channels of respective orders would be lesser than that of pore-throats and followed by pore-bodies of corresponding orders. 


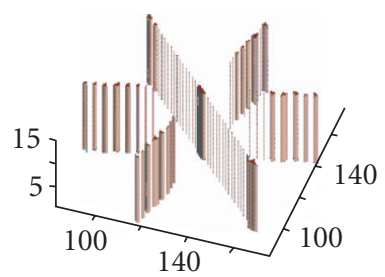

(a)

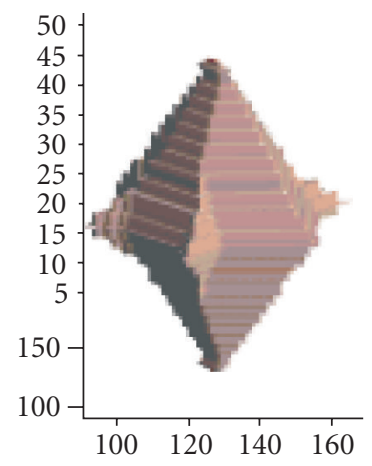

(d)

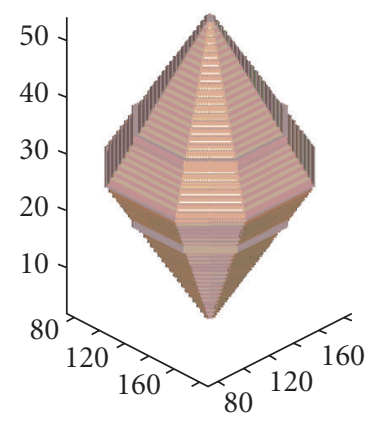

(g)

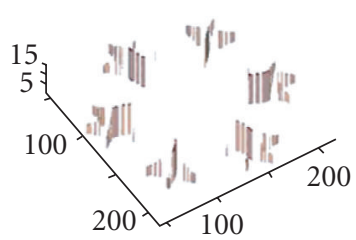

(b)

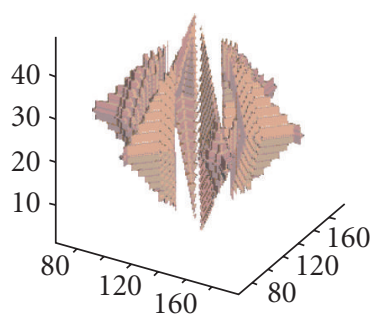

(e)

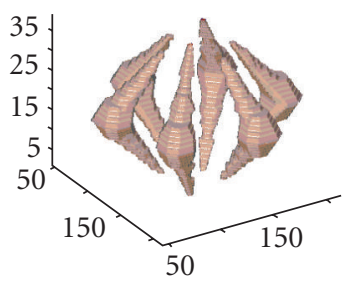

(h)

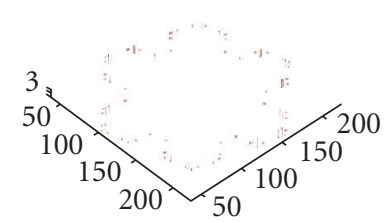

(c)

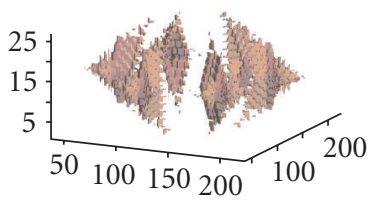

(f)

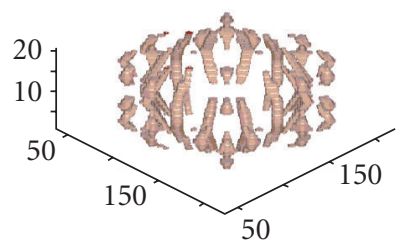

(i)

Figure 4.3. Orderwise (a)-(c) pore-channels, (d)-(f) pore-throats, and (g)-(h) pore-bodies.

4.3. Ordering scheme of morphological quantities. In order to properly designate 3D pore-channels and -throats with orders, 3D pore-bodies of various categories need to be first designated with orders. The pore-body-order designation in $3 \mathrm{D}$ is done based on a set of (4.7). The channel(s) or throat(s) that are contained in the $n$ th-order porebody(ies) are designated as $n$ th-order pore-channel(s) and -throat(s). In turn, whatever the channel and throat portions intersect with, first-order pore-bodies are designated as first-order channels and throats. Nth-level channel, throat, and bodies (4.3)-(4.7) are considered as order 1 , and $N$-1st-level pore-channel, -throat, and -bodies are considered 
as order 2 and so on. In this fashion, the pore-channels, -throats, and -bodies are designated with respective orders. This order designation is mathematically depicted as

$$
\begin{aligned}
& {[\operatorname{PTN}(G)] \bigcap\left[\left(\operatorname{PBN}^{n}\right)(G)\right]=\operatorname{PTN}^{n}(G),} \\
& {[\operatorname{PCN}(G)] \bigcap\left[\left(\operatorname{PBN}^{n}\right)(G)\right]=\operatorname{PCN}^{n}(G) .}
\end{aligned}
$$

In other words, morphologically, the relationship is shown as follows:

$$
\left[\operatorname{PCN}^{n}(G)\right] \subset\left[\operatorname{PTN}^{n}(G)\right] \subset\left[\operatorname{PBN}^{n}(G)\right] \subset[G], \quad \text { where } n=1,2, \ldots, N
$$

4.4. Estimation of orderwise pore $3 \mathrm{D}$ morphological quantities. Volumes of orderwise pore-channels, -throats, and -bodies connected across slices as explained in (4.10)-(4.12) and that are fragmented from each 2D slice of pore-image are also estimated to provide graphical relationships,

$$
\begin{gathered}
V\left[\operatorname{PCN}^{1}(G)\right]=\sum_{j=1}^{55} A\left[\operatorname{PCN}_{N}\left(M^{j}\right)\right], V\left[\operatorname{PCN}^{2}(G)\right]=\sum_{j=1}^{55} A\left[\operatorname{PCN}_{N-1}\left(M^{j}\right)\right], \ldots, \\
V\left[\operatorname{PCN}^{N}(G)\right]=\sum_{j=1}^{55} A\left[\operatorname{PCN}_{1}\left(M^{j}\right)\right], \\
V\left[\operatorname{PTN}^{1}(G)\right]=\sum_{j=1}^{55} A\left[\operatorname{PTN}_{N}\left(M^{j}\right)\right], V\left[\operatorname{PTN}^{2}(G)\right]=\sum_{j=1}^{55} A\left[\operatorname{PTN}_{N-1}\left(M^{j}\right)\right], \ldots, \\
V\left[\operatorname{PTN}^{N}(G)\right]=\sum_{j=1}^{55} A\left[\mathrm{PTN}_{1}\left(M^{j}\right)\right], \\
V\left[\operatorname{PBN}^{1}(G)\right]=\sum_{j=1}^{55} A\left[M_{1}^{j} \circ N S\right], V\left[\operatorname{PBN}^{2}(G)\right]=\sum_{j=1}^{55} A\left[M_{2}^{j} \circ N S\right], \ldots, \\
V\left[\operatorname{PBN}^{N}(G)\right]=\sum_{j=1}^{55} A\left[M_{N}^{j} \circ N S\right] .
\end{gathered}
$$

In other words, the total volumes of pore-channel, -throats, and -bodies can be, respectively, derived from $V[\operatorname{PCN}(G)]=\sum_{i=1}^{N} V\left[\operatorname{PCN}^{i}(G)\right], V[\operatorname{PTN}(G)]=\sum_{i=1}^{N} V\left[\operatorname{PTN}^{i}(G)\right]$, and $V[\operatorname{PBN}(G)]=\sum_{i=1}^{N} V\left[\operatorname{PBN}^{i}(G)\right]$.

Further volume fractions are computed for the order-distributed channels, throats, and bodies (Table 4.1). The order versus number and their corresponding voxel counts are plotted as graphs and complexity measures are estimated for the model fractal pore considered demonstrating the framework.

\section{Relationships between pore morphological quantities: results and discussion}

Three-dimensional volumes for model pore and corresponding orderwise channels, throats, and bodies are computed by stacking the slices that are generated via erosion 


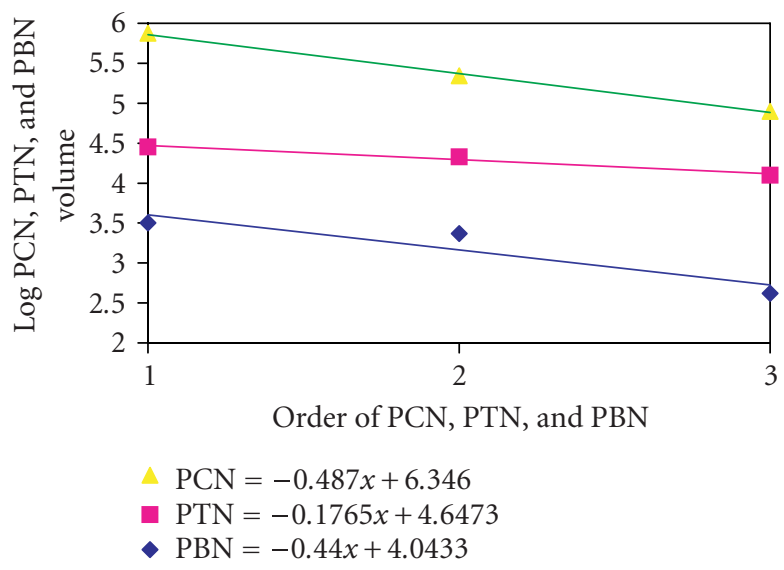

Figure 5.1. Distribution of pore-channel, -throat, and -body of fractal pore.

cycles of pore regions and their slicewise morphologic quantities extracted at respective phases. Total volumes of pore-channel, pore-throats, and pore-bodies estimated in voxels with slice thickness of one voxel, respectively, include 5938, 66520, and 1109016. It is obvious that these values of pore-bodies are higher followed by pore-throats and porechannels further supporting the fact that pore-channel network is subset of pore-throat network that is subset of pore-body network. This is the basic relationship by definition.

It is observed that the $\mathrm{PCN}_{n}(M) \subset \mathrm{PTN}_{n}(M) \subset \mathrm{PBN}_{n}(M)$. To characterize these geometrically significant pore morphologic features, we estimate the volumes occupied by the PCN, the PTN, and the PBN at respective subset levels. By estimating the volumes $V\left[\operatorname{PCN}_{n}(G)\right], V\left[\operatorname{PTN}_{n}(G)\right]$, and $V\left[\operatorname{PBN}_{n}(G)\right]$ in voxel units, we observe that these estimations are decreasing functions as depicted in the following: $V\left[P C N_{n}(G)\right]<$ $V\left[\mathrm{PTN}_{n}(G)\right]<V\left[\mathrm{PBN}_{n}(G)\right]$. To quantify the spatial complexities of the pore-channel, -throat, and -body networks, we plot logarithms of volumes of PCN, PTN, and PBN, and the corresponding designated orders (Figure 5.1). The volume fractions of orderwise pore-channels, -throats, and -bodies are plotted as functions of designated order number. The rate of change in the volume fractions across the orders is relatively rapid in the pore-bodies followed by pore-channels and pore-throats, as revealed from Figure 5.1.

The linear plots yield best fit coefficients, respectively, $0.49,0.18$, and 0.44 . These values are dependent on the shape of structuring element employed to decompose pore into channels, throats, and bodies. This shape dependency provides an insight further to relate empirically between the scale independent but shape-dependent power laws and other effective properties of complex porous media.

\section{Conclusions}

We presented a morphology-based framework to decompose the pore structure into pore-channels, -throats, and -bodies of various orders which facilitate to compute their accurate size distribution functions. We documented that an accurate pore morphometry 
can be carried out, once pore space has been properly decomposed into pore-channels, -throats, and -bodies of various orders. This approach is in general useful for analyzing, understanding geometrical properties, and relating them with physical properties. As the choice of template influences their spatial patterns, this study opens a way to understand important shape-dependent topologic properties of porous media. We hypothesize that these morphologically significant decomposed pore features at multiple scales can be related with bulk material properties. One can also show new results by employing multiscale morphological transformations that can be treated as a transformation meant for showing systematic variations in porosity. Sparse and intricate PCN, PTN, and PBN would be obvious, respectively, from simple and complex pore spaces. Variations in decomposed pore morphologic parameters across slices in the tomographic data (e.g., Fontainebleau sandstone) further provide potentially valuable insights. This entire approach can be extended to any 3D pore-image, that is constructed by stacking the $2 \mathrm{D}$ slices/tomographic images, to isolate orderwise fragmented pore-channels, throats, and bodies with appropriate connectivity across slices. The full potential and limitations of this approach have yet to be fully explored. If the physicochemical reasons governing porosity of a material are understood, then properties measured/calculated (such as channel connectivity, pore-body distribution, pore-throat distribution) can be manipulated accordingly for practical/technological applications. However, the different steps accomplished from this investigation pave a way to relate the statistically derived properties with physical properties. In our follow-up study, we apply this framework on Fontainebleau sandstone sample to further relate the computed complexity measures with that of bulk properties that are well documented.

\section{Symbols, notations, and nomenclature}

$R^{n}=$ Euclidean $n$-dimensional space.

$Z^{n}=$ Discrete $n$-dimensional space.

$M^{j}=j$ th slice of pore-image.

$n S=n$th size of structuring element.

$\hat{S}=$ Transpose of structuring element.

$S=\hat{S}=$ Symmetric structuring element.

$N S=$ Largest size of structuring element that fits into pore-image.

$\operatorname{PBN}^{n}\left(M^{j}\right)=n$th size of pore-body in $j$ th slice of pore-image.

$\operatorname{PTN}^{n}\left(M^{j}\right)=n$th size of pore-throat in $j$ th slice of pore-image.

$\operatorname{PCN}^{n}\left(M^{j}\right)=n$th level of pore-channel in $j$ th slice of pore-image.

$G=$ Stack of all slices of pore-image $\left(M^{j}\right), j=1,2, \ldots, N$.

$\cup, \cap, \backslash=$ Logical union, logical intersection, and logical difference.

$M \ominus S$ Morphological erosion of $M$ with respect to $S$.

$M \oplus S=$ Morphological dilation of $M$ with respect to $S$.

$M \circ S=$ Morphological opening of $M$ with respect to $S$.

$M_{i}^{j}=i$ th-level subtracted portion of $j$ th slice of pore-image.

$\operatorname{PCN}_{N}\left(M^{j}\right)=N$ th-level channel subset(s) of $j$ th slice of pore-image. 
$\operatorname{PTN}_{N}\left(M^{j}\right)=N$ th-level throat subset(s) of $j$ th slice of pore-image.

$\left(M_{i}^{j} \circ N S\right)=N$ th-level pore-body(ies) from $i$ th-level subtracted portion of $j$ th slice of pore-image.

$\operatorname{PCN}^{N}(G)=$ Stack of first-order channel subsets decomposed from all slices of pore-image.

$\operatorname{PTN}^{N}(G)=$ Stack of first-order pore-throat(s) decomposed from all slices of pore-image.

$\operatorname{PBN}^{N}(G)=$ Stack of first-order pore-body(ies) decomposed from all slices of pore-image.

\section{Acknowledgments}

We gratefully acknowledge the comments and suggestions on the earlier version by Gabor Korvin, M. Prodanovic, and W. B. Lindquist, which improved the manuscript.

\section{References}

[1] C. H. Arns, M. A. Knackstedt, W. Val Pinczewski, and W. B. Lindquist, Accurate estimation of transport properties from microtomographic images, Geophysical Research Letters 28 (2001), no. $17,3361-3364$.

[2] M. J. Beran, Statistical Continuum Theories, John Wiley \& Sons, New York, 1968.

[3] M. A. Celia, P. C. Reeves, and L. A. Ferrand, Recent advances in pore scale models for multiphase flow in porous media, Reviews of Geophysics 33 (1995), no. S1, 1049-1058.

[4] D. A. Coker and S. Torquato, Extraction of morphological quantities from a digitized medium, Journal of Applied Physics 77 (1995), no. 12, 6087-6099.

[5] D. A. Coker, S. Torquato, and J. H. Dunsmuir, Morphology and physical properties of Fountainbleau sandstone via a tomographic analysis, Journal of Geophysical Research 101 (1996), no. B8, 17497-17506.

[6] C. Lantuéjoul, La squelettization et son application aux mesures topologuiques des mosaiques polycrstallines, Ph.D. dissertation, School of Mines, Paris, 1978.

[7] W. B. Lindquist, Network flow model studies and 3D pore structure, Fluid Flow and Transport in Porous Media: Mathematical and Numerical Treatment (South Hadley, MA, 2001), Contemp. Math., American Mathematical Society, Rhode Island, 2002, pp. 355-366.

[8] W. B. Lindquist, S.-M Lee, D. A. Coker, K. W. Jones, and P. Spanne, Medial axis analysis of void structure in three-dimensional tomographic images of porous media, Journal of Geophysical Research 101 (1996), no. B4, 8297-8310.

[9] W. B. Lindquist and A. Venkatarangan, Investigating 3D geometry of porous media from high resolution images, Physics and Chemistry of the Earth 24 (1997), no. 7, 593-599.

[10] W. B. Lindquist, A. Venkatarangan, J. Dunsmuir, and T.-F. Wong, Pore and throat size distributions measured from synchrotron X-ray tomographic images of Fountainbleau sandstones, Journal of Geophysical Research 105 (2000), no. B9, 21509-21528.

[11] B. Lu and S. Torquato, n-point probability functions for a lattice model of heterogeneous media, Physical Review B 42 (1990), no. 7, 4453-4459.

[12] Lineal-path functions for random heterogeneous materials, Physical Review A 45 (1992), no. 2, 922-929.

[13] G. Matheron, Elements pour une Theorie des Milieux Poreux, Masson, Paris, 1967.

[14] R. Orbach, Dynamics of fractal networks, Science 231 (1986), 814-819.

[15] E. Perrier, N. Bird, and M. Rieu, Generalizing the fractal model of soil structure: the pore-solid fractal approach, Geoderma 88 (1999), no. 3-4, 137-164. 
[16] S. Prager, Interphase transfer in stationary two-phase media, Chemical Engineering Science 18 (1963), no. 4, 227-231.

[17] J. Serra, Image Analysis and Mathematical Morphology, Academic Press, London, 1982.

[18] D. Silin, G. Jin, and T. W. Patzek, Robust determination of pore space morphology in sedimentary rocks, SPE Paper \# 84296, 2003.

[19] L. L. Teo, P. Radhakrishnan, and B. S. Daya Sagar, Morphological decomposition of sandstone pore-space: fractal power-laws, Chaos, Solitons \& Fractals 19 (2004), no. 2, 339-346.

[20] S. Torquato, Microstructure characterization and bulk properties of disordered two-phase media, Journal of Statistical Physics 45 (1986), no. 5-6, 843-873.

[21] _ Modeling of physical properties of composite materials, International Journal of Solids and Structures 37 (2000), no. 1-2, 411-422.

[22] S. Torquato and B. Lu, Chord-length distribution function for two-phase random media, Physical Review E 47 (1993), no. 4, 2950-2953.

[23] D. L. Turcotte, Fractals and fragmentation, Journal of Geophysical Research 91 (1986), no. B2, 1921-1926.

[24] H. J. Vogel and K. Roth, Quantitative morphology and network representation of soil pore structure, Advances in Water Resources 24 (2001), no. 3-4, 233-242.

Lay Lian Teo: Faculty of Engineering and Technology, Multimedia University, Melaka Campus, Jalan Ayer Keroh Lama, Melaka 75450, Malaysia

E-mail address: 1lteo@mmu.edu.my

B. S. Daya Sagar: Faculty of Engineering and Technology, Multimedia University, Melaka Campus, Jalan Ayer Keroh Lama, Melaka 75450, Malaysia

E-mail address: bsdaya.sagar@mmu.edu.my 


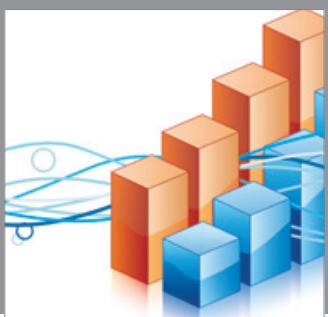

Advances in

Operations Research

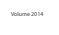

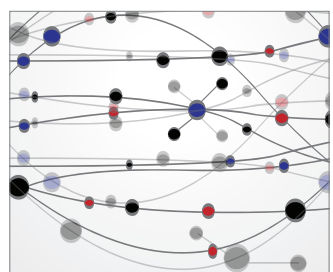

\section{The Scientific} World Journal
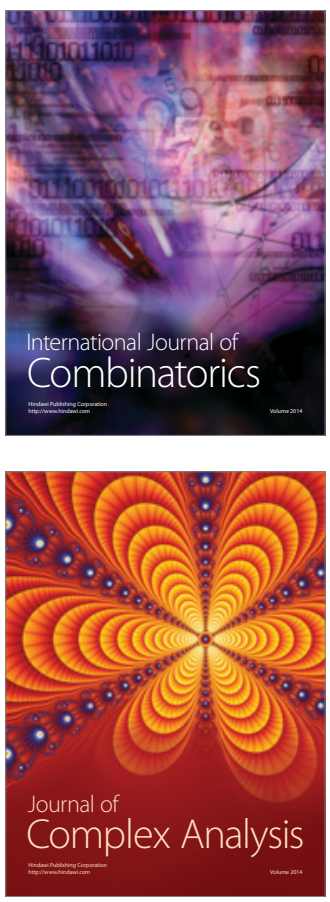

International Journal of

Mathematics and

Mathematical

Sciences
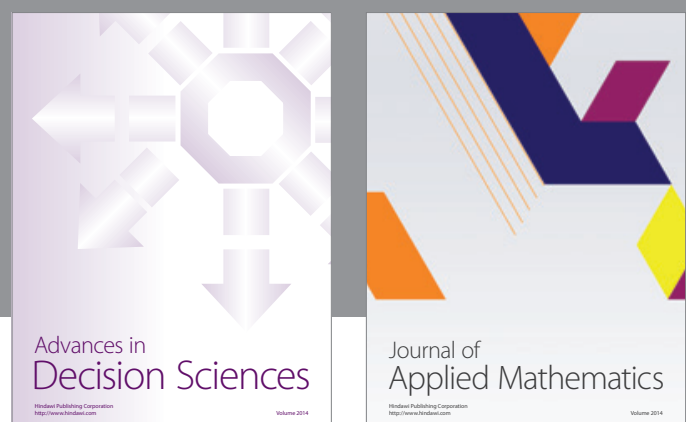

Journal of

Applied Mathematics
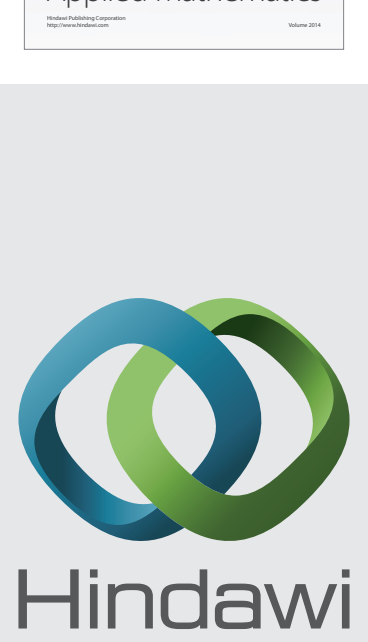

Submit your manuscripts at http://www.hindawi.com
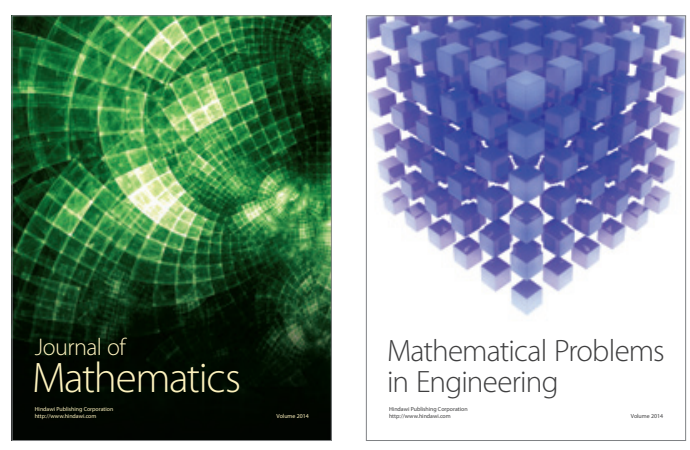

Mathematical Problems in Engineering
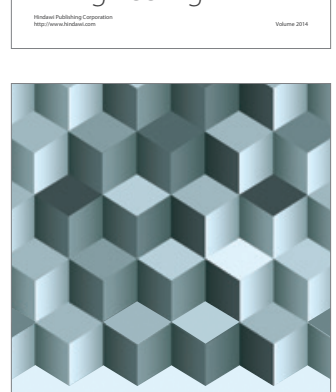

Journal of

Function Spaces
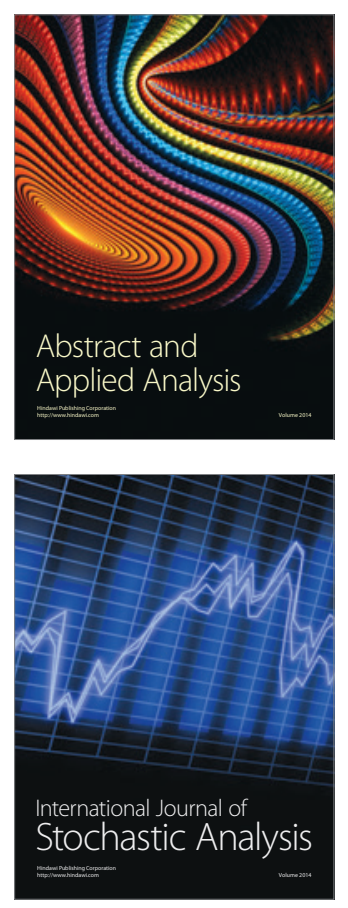

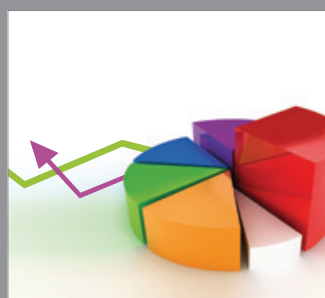

ournal of

Probability and Statistics

Promensencen
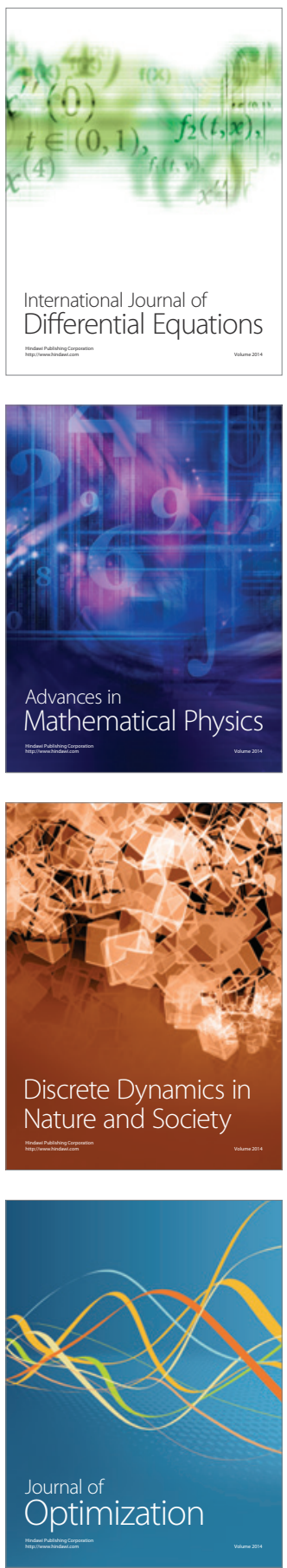